\section{Gastroenterology Service}

\section{OTU-028 BENCHMARKING OF ACTIVITY, PROCESS AND OUTCOME OF EMERGENCY ADMISSION FOR ULCERATIVE COLITIS ACROSS ENGLISH HOSPITALS}

\author{
${ }^{1}$ Mustafa Shawihdi*, ${ }^{1}$ Susanna Dodd, ${ }^{1}$ Duncan Appelbe, ${ }^{1}$ Pete Dixon, ${ }^{1}$ Ruth Grainger, \\ ${ }^{3}$ Fraser Cummings, ${ }^{2}$ Stuart Bloom, ${ }^{1}$ Keith Bodger*. ${ }^{1}$ University of Liverpool, Liverpool, UK; \\ ${ }^{2}$ University College London Hospital, London, UK; ${ }^{3}$ University Hospitals Southampton, \\ Southampton, UK
}

\subsection{6/gutjnl-2018-BSGAbstracts.442}

Introduction The UK IBD Registry aims to make information work better for patients, clinical teams and the NHS. As part of the benchmarking reports provided to participating Trusts, we have developed organisational level metrics from routinely collected hospital episode statistics (HES) data - working with front-line teams to iteratively develop reports with feedback on content and local face-validity. We report national-level findings and institutional variation in activity, process and outcome of emergency care for UC.

Methods Admitted patient care data for English hospitals were analysed, identifying all-cause admissions for patients with UC and constructing algorithms to identify emergency activity, track process and outcome for UC-specific emergency admissions (UC-Em-Ad), including in-hospital death (I-H-D) and emergency surgery (Em-Surg), all-cause 30 day readmission (30D-RA) and twelve month outcome. Reports containing 5 year national and local trends and cumulative 5 year performance were distributed to sites in Dec 2017. This analysis summarises selected data for 133 Trusts present in all fiscal years $(11 / 12$ to $15 / 16)$.

Results Nationally, there were 31,371 UC-Em-Ad (2 65799 bed days; median LoS 6 days; 22809 patients; mean age 40 years; male 50\%; additional coded co-morbidities in $23 \%)$ with 1451 Em-Surg $(4.62 \%=$ crude surgery rate; mean age 44 years; male 56.1\%), 324 I-H-D $(1.03 \%=$ crude mortality rate; mean age 76 years; $67 \%$ had additional coded co-morbidities; only $16 \%$ of deaths were post-surgery), 4916 30D-RA $(15.7 \%=$ crude readmission rate). At Trust level, mean $(95 \%$ limits) for indirectly standardised rates were: I-H-D $1.03 \% \quad(0.90 \%-1.15 \%)$, Em-Surg $4.79 \%$ (4.31\%-5.27\%), 30D-RA 15.55\% (15.0\%-16.1\%). Few outliers were identified and none consistently over time, with no significant trends identified for volume-outcome relationships. Funnel plots and regression analyses will be presented.

Conclusions These data provide real-world insights into processes and outcomes of emergency care for UC across England in the last five years, with a series of metrics to support both national and local quality improvement efforts. Linkages between HES and local Registry data offers potential to validate, refine and extend these benchmarking metrics.

Funding Crohn's and Colitis UK.

\section{OTU-029 FAECAL IMMUNOCHEMICAL TESTS (FIT) FOR SURVEILLANCE AFTER SCREENING AND POLYPECTOMY: AN ACCURACY AND EFFICIENCY STUDY}

${ }^{1}$ Emma Robbins*, 'Kate Wooldrage, ${ }^{1}$ Eilidh MacRae, 'lain Stenson, 'Bhavita Patel, ${ }^{1}$ Kevin Pack, ${ }^{2}$ Carolyn Piggott, ${ }^{2}$ Sheena Pearson, ${ }^{2}$ Julia Snowball, ${ }^{3}$ Stephen Duffy, ${ }^{2}$ Stephen Halloran, 'Wendy Atkin, ${ }^{1}$ Amanda Cross. ${ }^{1}$ Cancer Screening and Prevention Research Group, Imperial College London, London; ${ }^{2}$ Bowel Cancer Screening Programme Southern Hub, Guildford; ${ }^{3}$ Centre for Cancer Prevention, Wolfson Institute of Preventive Medicine, Queen Mary University, London

\subsection{6/gutjnl-2018-BSGAbstracts.443}

Introduction Individuals at intermediate-risk for colorectal cancer (CRC) following adenoma removal within the English Bowel Cancer Screening Programme (BCSP) are invited for three-yearly surveillance colonoscopy. Given the invasive nature of colonoscopy and scarcity of endoscopy resources, there is a need for an alternative surveillance method. We aimed to determine whether annual testing with the faecal immunochemical test (FIT) is an effective alternative.

Methods Individuals aged 60-72 years and scheduled for surveillance following removal of intermediate-risk adenomas were recruited within the BCSP from January 2012 to December 2013. Quantitative FIT (OC-Sensor, Eiken) was offered at one, two, and three years post-polypectomy. Invitees who returned a completed consent form and an analysable FIT at Round 1 were included. Participants testing positive $(\geq 40 \mu \mathrm{g}$ haemoglobin $(\mathrm{Hb}) / \mathrm{g}$ faeces) at Rounds 1 or 2 were offered early colonoscopy and were not invited to further FIT rounds. All other participants were offered the routine three-year surveillance colonoscopy. Diagnostic accuracy for CRC and advanced adenomas (AAs: adenomas $\geq 10 \mathrm{~mm}$, with tubulovillous or villous histology, or high grade dysplasia) was calculated at each round, using colonoscopy as the reference standard. We estimated diagnostic accuracy with lower haemoglobin thresholds and multiple rounds.

Results Of 8008 invitees, 5946 (74\%) consented and returned an analysable FIT at Round 1. Uptake of FIT was higher (97\%) in Rounds 2 and 3. FIT positivity decreased by round, from $6 \%$ to $4 \%$ in Rounds 1 to 3 . In total, 26 participants were diagnosed with CRC and 443 with AAs. At $40 \mu \mathrm{g} / \mathrm{g}$, sensitivity and specificity of the first FIT were, respectively, $31 \%$ and $94 \%$ for CRC and $18 \%$ and $95 \%$ for AAs. Sensitivities for CRC and AAs were higher, and specificities lower, with lower thresholds and multiple rounds. At $10 \mu \mathrm{g} / \mathrm{g}$, the programme sensitivity and specificity of three rounds were, respectively, $85 \%$ and $71 \%$ for CRC and $57 \%$ and $73 \%$ for AAs.

Conclusions Annual low threshold FIT achieved relatively high sensitivity for CRC over three years. If this strategy replaced three-yearly surveillance colonoscopy, the number of colonoscopies could potentially be reduced by $70 \%$. However, sensitivity for AAs was limited. Further research is needed to consider the implications for clinical practice of missing CRCs and AAs with FIT-based surveillance. 